\title{
Single photon emission computed
}

\section{tomography myocardial perfusion imaging in patients with moderate to severe} psoriasis

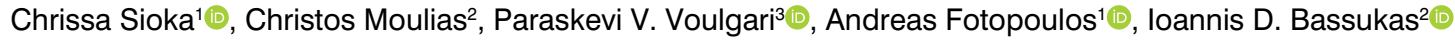 \\ ${ }^{1}$ Department of Nuclear Medicine, Medical School, University Hospital of loannina, Greece \\ 2Department of Skin and Venereal Diseases, Medical School, University Hospital of loannina, Greece \\ ${ }^{3}$ Rheumatology Clinic, Department of Internal Medicine, Medical School, University of Ioannina, Greece
}

[Received 24 VI 2021; Accepted 09 VI 2021]

\begin{abstract}
Background: Psoriasis is a chronic inflammatory disorder with an increased risk for coronary artery disease (CAD). This retrospective study aimed to evaluate the rate of myocardial ischaemia in patients with psoriasis subjected to myocardial perfusion imaging (MPI).
\end{abstract}

Material and methods: Twelve patients with moderate to severe psoriasis that had MPI were compared to 395 MPIs randomly retrieved from our MPIs pool data. All patients had a [ ${ }^{99 \mathrm{~m} T c]}$ tetrofosmin stress - rest single-photon emission computer tomography ([99mTc]SPECT). Summed difference scores (SDS) were calculated for stress (SSS), rest (SRS) and their difference $(\mathrm{SDS}=\mathrm{SSS}-\mathrm{SRS})$.

Results: There was no significant difference in the frequency of abnormal MPI SPECT outcomes between patients with vs. without psoriasis $(6 / 12$ vs $214 / 395$ respectively; $p=0.778)$. From the evaluation of SSS, SRS and SDS, only the SDS scores of inadequately compensated resting perfusion defects were significantly lower in patients with psoriasis $(p=0.012)$.

Conclusions: Patients with moderate-to-severe psoriasis had a similar rate of abnormal SSS scans compared to control patients. However, the SDS scans were significantly lower in patients with psoriasis indicating compromised reversibility of resting perfusion defects. Larger controlled studies are needed to verify these observations.

KEY words: psoriasis; myocardial perfusion imaging; SPECT; myocardial ischaemia; perfusion defects

Nucl Med Rev 2021; 24, 2: 46-50

\section{Introduction}

Psoriasis is a chronic, recurrent, multifactorial inflammatory skin disorder with a complex co-morbidities profile, which includes increased coronary artery disease (CAD) risk [1]. The higher prevalence of cardiovascular risk factors is translated into a higher CAD burden [2], the higher lifetime risk of a major adverse

Correspondence to: Andreas Fotopoulos

Department of Nuclear Medicine, Medical School,

University Hospital of loannina, Greece

e-mail: professor.fotopoulos@yahoo.com cardiovascular event (MACE) and a higher cardiovascular mortality rate, however, the latter only in patients with severe disease and/or psoriatic arthritis [3].

Whenever feasible, coronary angiography $(C A)$ remains the gold standard for the diagnosis of CAD. Alternatively, non-invasive cardiovascular imaging methods are important for the prediction of future MACE in patients with CA contraindications. Among them, myocardial perfusion imaging (MPI) single photon emission computed tomography (SPECT) is probably the modality with the highest impact to detect a silent myocardial ischaemic region [4]. To date, MPI data has been only occasionally reported in patients with psoriasis $[5,6]$. This retrospective study aimed to evaluate the myocardial status in patients with moderate-to-severe psoriasis utilizing MPI. 


\section{Material and methods}

By comparing the medical records of the Nuclear Medicine and Dermatology Departments we identified 12 MPIs study outcomes in 12 patients with a history of moderate-to-severe psoriasis. Since the prevalence rate of all psoriasis cases in the reference population is approximately $3 \%$ [7], we additionally retrieved randomly MPI results of 395 patients without psoriasis which formed the control group independent of their medical history, cardiac or other diseases. Thus, this yielded the study population of 407 patients, which included approximately $3 \%(12 / 407)$ of the patient group and $97 \%$ (395/407) of the control group. The assumption was that randomly selected that large control group of patients with other medical conditions could better balance any variations in patient characteristics between the 2 groups.

All MPIs (inpatients and control individuals) were performed after requisition by a cardiologist for a variety of reasons, using a 1-day imaging protocol according to published guidelines [8], with stress protocol consisted either of a dynamic exercise - Bruce protocol treadmill exercise test or a pharmacological exercise with dipyridamole or dobutamine. The injected dose was $8 \mathrm{mCi}$ [99mTc] tetrofosmin at stress and $20 \mathrm{mCi}\left[{ }^{99 \mathrm{~m}} \mathrm{Tc}\right]$ tetrofosmin at rest. Forty minutes later, the images were acquired via a $90^{\circ}$-angled dual-head camera, using a collimator with 64 stops and $25 \mathrm{~s}$ per projection over a $180^{\circ}$ arc. Subsequently, the images were reconstructed without attenuation correction as previously reported [9].

Images were visually evaluated by two nuclear medicine specialists, applying a 5-point severity scoring scale from normal $($ score $=0)$ to absent $($ score $=4)$ perfusion to a 17 segments polar myocardium map as previously reported $[10,11]$.

Summed stress scores (SSS), summed rest scores (SRS) and summed difference scores (SDS) was valued for semiquantitative visual analysis. Summed scores over the 17 myocardium segments (range: 0-64) were evaluated separately for stress [SSS reflects both stress-induced and resting perfusion defects (i.e., ischaemia + infarct)] and rest (SRS-resting perfusion defects (i.e., reflecting scar/hibernating myocardium or artifact]) images. In addition, their difference [SDS = SSS - SRS, a measure of reversibility in response to exercise or pharmacologic stress, (i.e., ischaemia)], was additionally calculated. SSS scores $\geq 4$ were considered to indicate myocardial ischaemia - ('pathologic' MPI), graded further as mild $(4 \leq \mathrm{SSS}<9)$, moderate $(9 \leq \mathrm{SSS}<14)$ and severe for $S S S \geq 4$ [12]. Comparisons of traits of interest between patients with vs. without psoriasis were quantified by Fisher's exact, $\chi^{2}$ - and Mann-Whitney U- tests. The impact of sex (male, female), age ('elderly', i.e. > 65, versus younger patients) and psoriasis (yes, no) on the MPI scores was inferred with 'generalized linear' and for the dichotomous outcome 'pathologic' MPI with logistic regression models respectively. All statistical tests were calculated with the SPSSv26 software at a significance level $p<0.05$.

\section{Results}

Relevant demographic and laboratory findings of the 12 psoriasis patients [ 6 men and 6 women; mean age: 70.7 years; psoriasis area and severity index (PASI) score: 19.2] are summarized in Supplementary Table 1. Patients with psoriasis were significantly older at the time of MPI (in average about 10 years, $p=0.001$,
Mann-Whitney U-test; Tab. 2) Psoriatic patients had also increased inflammation parameters, C-reactive protein (CRP; average: 10.4) and erythrocyte sedimentation rate (ESR; average: 39.1), which reflect disease activity. In addition, most patients with psoriasis had traditional risk factors for CAD such as increased cholesterol levels and diabetes mellitus and some of them a history of cardiac morbidity (Tab. 1).

There was no significant difference in the frequency of abnormal MPI outcomes between patients with vs. without psoriasis $(6 / 12$ vs $214 / 395$ respectively; $p=0.778$, Fisher's exact test) or the distributions of the different MPI assessed degrees of myocardial ischaemia ( $p=0.756 ; \chi^{2}$-test). From the 3 parameters of the MPI evaluation (SSS, SRS and SDS), only the SDS scores differed between patients with and without psoriasis, with SDS being significantly lower in patients with psoriasis $(p=0.012$, Mann-Whitney U-test; Tab. 2 and Fig. 1). Moreover, the above impact of psoriasis on the SDS scores was substantiated further by analysing the impact of age, sex and psoriasis on the MPI scores with regression

Table 1. Demographic, clinical and laboratory characteristics of $\mathrm{n}=12$ patients with psoriasis

\begin{tabular}{|c|c|c|c|}
\hline & \multicolumn{3}{|c|}{ Range } \\
\hline & Mean $\left(\mathrm{SEM}^{1}\right)$ & Min & $\operatorname{Max}$ \\
\hline Age (year) & $70.7(1.8)$ & 56 & 78 \\
\hline Sex (number) & 6 Male / 6 Female & & \\
\hline \multicolumn{4}{|l|}{ Psoriasis characteristics } \\
\hline PASI & $19.2(2.7)$ & 10 & 35 \\
\hline Psoriatic arthritis (number) & $1 / 12$ & & \\
\hline Acrodermatitis continua (number) & $1 / 12$ & & \\
\hline $\operatorname{ESR}^{1}(\mathrm{~mm} / \mathrm{h})$ & $39.1(5.5)$ & 19 & 72 \\
\hline $\mathrm{CRP}^{1}$ (mg/l, normal values: 0-6) & $10.4(1.8)$ & 2 & 20 \\
\hline Elevated CRP (number) & $8 / 12$ & & \\
\hline \multicolumn{4}{|l|}{ Cholesterol (mg/dl) } \\
\hline Total cholesterol & $198.7(17.9)$ & 87 & 267 \\
\hline $\mathrm{LDL}^{1}$ & $116.7(15.0)$ & 26 & 176 \\
\hline $\mathrm{HDL}^{1}$ & $42.0(3.1)$ & 25 & 56 \\
\hline LDL / HDL & $2.7(0.3)$ & 1.0 & 3.6 \\
\hline Triglycerides & $205.3(16.3)$ & 109 & 255 \\
\hline Glucose (mg/dl) & $168.4(20.2)$ & 92 & 308 \\
\hline Psoriasis treatment (number) & $12 / 12$ & & \\
\hline Topical psoriasis treatment (number) & $12 / 12$ & & \\
\hline $\begin{array}{l}\text { Systemic psoriasis treatment } \\
\text { (number) }\end{array}$ & $12 / 12$ & & \\
\hline Systemic per os² (number) & $10 / 12$ & & \\
\hline Biological $^{3}$ (number) & $10 / 12$ & & \\
\hline \multicolumn{4}{|l|}{$\mathrm{CVD}^{1}$} \\
\hline Myocardial infarction (number) & $2 / 12$ & & \\
\hline Heart insufficiency (number) & $1 / 12$ & & \\
\hline Aorta insufficiency (number) & $2 / 12$ & & \\
\hline Atrial fibrillation (number) & $2 / 12$ & & \\
\hline
\end{tabular}

${ }^{1}$ Abbreviations. CRP - C-reactive protein, CVD: cardiovascular disease, ESR — erythrocyte sedimentation rate, $\mathrm{HDL}$ — high-density lipoprotein, LDL — lowdensity lipoprotein, SEM — standard error of the mean. ${ }^{2}$ acitretin, apremilast ${ }^{3}$ Adalimumab, efalizumab, etanercept, infliximab, secukinumab, ustekinumab 
Table 2. Comparison of age at SPECT MPI conduction and SPECT MPI myocardial ischemia scores between patients with psoriasis and controls (mean [SEM]; p-values: Mann-Whitney U-test)

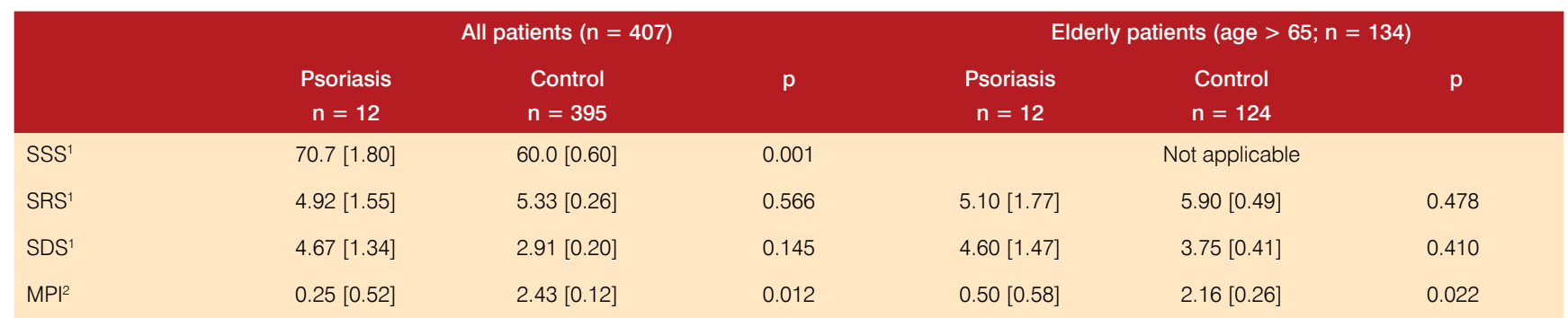

ISPECT MPI ischaemia score: generalized linear model; ${ }^{2}$ myocardial ischaemia (pathologic MPI): logistic regression model; MPI — pathologic myocardial perfusion by MPI SPECT (SSS $\geq 4$ ); SDS — Ischaemia reversibility scores, SDS = SSS - SRS; SEM — Standard Error of the Mean; SRS — Summed Rest Score; SSS — Summed Stress Score; SRS: Summed Rest Score; SDS — Ischemia reversibility scores, SDS = SSS-SRS; MPI— pathologic myocardial perfusion by MPI SPECT (SSS $\geq 4$ ), SEM — Standard Error of the Mean
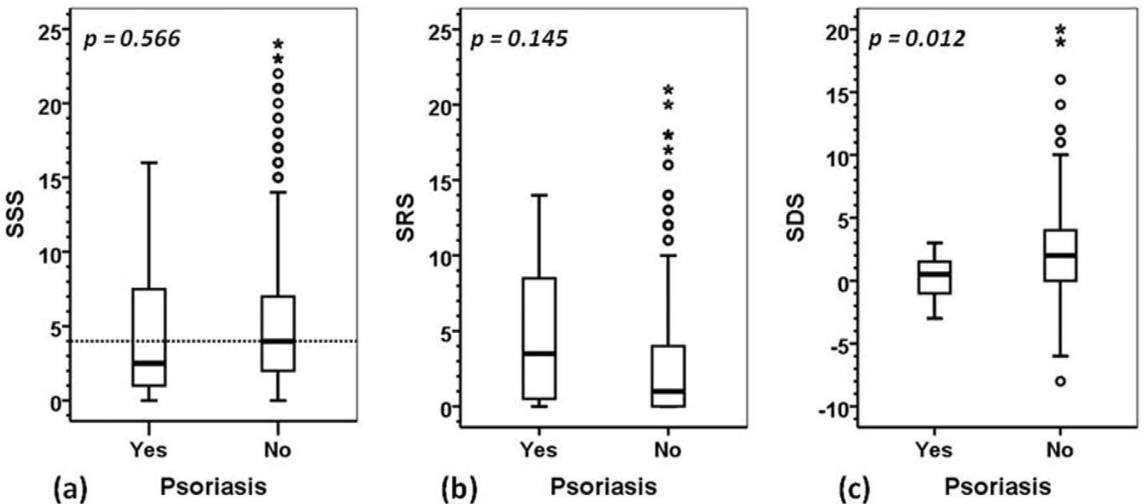

Figure 1. Box-plot diagrams of SPECT MPI summed scores of patients with vs. without psoriasis (p: significance; Mann-Whitney U-test);

A. SSS: ischaemia scores at stress. Dashed line: score limit for ischaemia; B. SRS: ischaemia scores at rest; C. SDS: scores of reversibility of stress ischaemia (SDS = SSS - SRS); MPI — myocardial perfusion imaging; SPECT — single photon emission computed tomography

models. Of these three factors, male sex was the most important and universal predictor of myocardial ischaemia (Tab. 3). However, psoriasis remains a significant independent predictor of the SDS $(p=0.023)$. Finally, older age was a significant predictor only of a higher degree of resting perfusion defects (SRS score; $p=0.016$ ).

\section{Discussion}

To the best of the authors' knowledge, this is the first report of the results of cardiologist's indicated MPI tests in patients with moderate-to-severe psoriasis. The main outcome of this study is a comparable prevalence of myocardial ischaemia (around $50 \%$ ) in patients with and without psoriasis, even though the former patients were significantly older at the time of the MPI conduction. However, the compromised myocardial oxygenation of the patients with moderate-to-severe psoriasis seems to be already established at a younger age. Employing MPI Zutt et al. [6], found a comparable rate of ischaemia (56\%) in 50 prospectively examined much younger psoriasis patients (average 49.3 years, i.e., about 20 years younger compared to the present cohort) with a similar disease burden. In this context, it is worth noting that Yalcin et al. [5], reported no pathologic MPI results in 28 much younger patients (average age: 41.2 years) with a rather mild psoriasis at the time of examination.
However, an association between cardiovascular disease and psoriasis is detected most consistently among patients with severe psoriasis. A systematic review and meta-analysis in which classification as severe psoriasis was based upon surrogate markers (e.g., a requirement for systemic treatment or hospital admission) found support for an increased risk for myocardial infarction, cardiovascular mortality, and stroke among patients with severe psoriasis [13]. In contrast to severe psoriasis, studies evaluating the relationship between milder disease and cardiovascular morbidity have yielded less consistent findings [14]. A longer duration of disease may be an additional risk factor for adverse cardiovascular events [15].

The rationale for a correlation between psoriasis and atherosclerotic disease is not well understood. Although the increased prevalence of main risk factors for cardiovascular disease, in patients with psoriasis likely contributes to the elevated risk for atherosclerosis, the role of chronic inflammation in the pathogenesis of both disorders may also be a key factor [16].

In a systematic review and meta-analysis that pooled the results of four studies, all-cause cardiovascular mortality was significantly greater in patients with severe psoriasis than in the general population [13]. A fifth study that reported a hazard ratio from the multivariate analysis offered additional support; the cohort study of approximately 3600 patients with moderate-to-severe psoriasis (defined as psoriasis treated with systemic therapy) and 
Table 3. Parameters (standard errors) of best-fit regression models of the effect of age ( $>65$ vs. $\leq 65$ ), sex (male vs. female) and psoriasis (yes vs. no) on the summed ischemia severity scores by SPECT MPI and on the diagnosis of a pathologic MPI test outcome ( $p=$ significance level).

\begin{tabular}{lccccccc} 
& Age & $p$ & Sex & $p$ & Psoriasis & $p$ \\
\hline SSS $^{1}$ & $0.46(0.48)$ & 0.340 & $5.12(0.46)$ & 0.000 & $-1.29(1.34)$ & 0.330 \\
SRS $^{1}$ & $0.91(0.38)$ & 0.016 & $3.79(0.36)$ & 0.000 & $0.81(1.04)$ & 0.436 \\
SDS $^{1}$ & $-0.45(0.34)$ & 0.180 & $1.35(0.32)$ & 0.000 & $-2.11(0.93)$ & 0.023 \\
MPI $^{2}$ & $-0.33(0.23)$ & 0.183 & $1.64(0.23)$ & 0.000 & $-0.41(0.65)$ & 0.534
\end{tabular}

SSS: Summed Stress ischemia Score; SRS: Summed Rest ischemia Score; SDS: Ischemia reversibility score (SDS = SSS - SRS); MPI: pathologic myocardial perfusion by MPI SPECT $(\mathrm{SSS} \geq 4)$. 1SPECT MPI ischemia score: Generalized linear model 2Myocardial ischemia (pathologic MPI): Logistic regression model

14,330 control patients without a history of psoriasis, found that patients with severe psoriasis were more likely than controls to die from the cardiac or cerebrovascular disease $[17,18]$.

Our present MPI findings demonstrated that older patients with moderate-to-severe psoriasis exhibited significantly less reversible ischaemia at rest, compared to the control group. In the light of these observations, the question arises whether therapies with a systemic anti-inflammatory impact, like anti-tumour necrosis factor-alpha antibodies agents, might attenuate and eventually prevent the evolution of myocardial ischaemia as a function of disease duration and severity and ultimately reduce the MACE risk [16].

\section{Conclusion}

In summary, we found a similar rate of myocardial ischaemia during stress in patients with moderate-to-severe psoriasis compared to control patients, but with less reversibility of ischaemia at rest. These findings are preliminary and their exact aetiology still unclear. The main limitation of this retrospective study is the small number of index patients. Larger, controlled studies are needed to verify our observations and inquire about the role of MPI studies in the evaluation of myocardial ischaemia in patients with psoriasis.

\section{Conflict of interest}

None declared.

\section{Disclosure}

None.

\section{Funding}

None.

\section{References}

1. Boehncke WH, Schön M. Psoriasis. The Lancet. 2015; 386(9997): 983-994, doi: 10.1016/s0140-6736(14)61909-7, indexed in Pubmed: 26025581.

2. Fernández-Armenteros JM, Gómez-Arbonés X, Buti-Soler M, et al. Psoriasis, metabolic syndrome and cardiovascular risk factors. A population-based study. J Eur Acad Dermatol Venereol. 2019; 33(1): 128-135, doi: 10.1111/jdv.15159, indexed in Pubmed: 29953676.
3. Raaby L, Ahlehoff $O$, de Thurah A. Psoriasis and cardiovascular events: updating the evidence. Arch Dermatol Res. 2017; 309(3): 225-228, doi: 10.1007/s00403-016-1712-1, indexed in Pubmed: 28213804.

4. Varadaraj G, Chowdhary GS, Ananthakrishnan R, et al. Diagnostic accuracy of stress myocardial perfusion imaging in diagnosing stable ischemic heart disease. J Assoc Physicians India. 2018; 66(8): 40-44, indexed in Pubmed: 31324083

5. Yalcin $H$, Balci DD, Ucar $E$, et al. Myocardial perfusion is preserved in patients with psoriasis without clinically evident cardiovascular disease. J Eur Acad Dermatol Venereol. 2009; 23(7): 798-802, doi: 10.1111/j.14683083.2009.03178.x, indexed in Pubmed: 19470047.

6. Zutt M, Rudolph H, Kaune KM, et al. Myocardial scintigraphy — a method for detecting cardiac comorbidity in psoriasis patients? J Dtsch Dermatol Ges. 2016: 14(10): 1007-1014, doi: 10.1111/ddg.12892, indexed in Pubmed: 27767272.

7. Bassukas I, Mavridou K, Evangelou T, et al. The prevalence of psoriasis among elderly individuals: more questions than answers. Ageing Research. 2011; 2(1): e1, doi: 10.4081/ar.2011.e1.

8. Arumugam P, Harbinson M, Reyes E, et al. Procedure guidelines for radionuclide myocardial perfusion imaging with single-photon emission computed tomography. Nucl Med Commun. 2013; 34(8): 813-826, doi: 10.1097/MNM.0b013e32836171eb, indexed in Pubmed: 23719150.

9. Fotopoulos A, Petrikis P, lakovou I, et al. The impact of depression and anxiety in prognosis of patients undergoing myocardial perfusion imaging with 99mTc tetrofosmin SPECT for evaluation of possible myocardial ischemia. Nucl Med Rev Cent East Eur. 2020; 23(2): 58-62, doi: 10.5603/NMR. a2020.0014, indexed in Pubmed: 33007091

10. Sioka C, Exarchopoulos T, Tasiou I, et al. Myocardial perfusion imaging with $(99 \mathrm{~m})$ Tc-tetrofosmin SPECT in breast cancer patients that received postoperative radiotherapy: a case-control study. Radiat Oncol. 2011; 6: 151, doi: 10.1186/1748-717X-6-151, indexed in Pubmed: 22067743.

11. Fotopoulos A, Papadimitropoulos K, Papadopoulos A, et al. Myocardial ischemia in female patients with rheumatoid arthritis assessed with single photon emission tomography-myocardial perfusion imaging. Nucl Med Rev Cent East Eur. 2019; 22(1): 8-13, doi: 10.5603/NMR.2019.0001, indexed in Pubmed: 31482536.

12. Giannopoulos S, Markoula S, Sioka $\mathrm{C}$, et al. Detecting myocardial ischemia with technetium-tetrofosmin myocardial perfusion imaging in ischemic stroke. Neurohospitalist. 2017; 7(4): 164-168, doi: 10.1177/1941874417704752, indexed in Pubmed: 28974994.

13. Samarasekera EJ, Neilson JM, Warren RB, et al. Incidence of cardiovascular disease in individuals with psoriasis: a systematic review and meta-analysis. J Invest Dermatol. 2013; 133(10): 2340-2346, doi: 10.1038/jid.2013.149, indexed in Pubmed: 23528816.

14. Dowlatshahi EA, Kavousi M, Nijsten T, et al. Psoriasis is not associated with atherosclerosis and incident cardiovascular events: the Rotterdam Study. J Invest Dermatol. 2013; 133(10): 2347-2354, doi: 10.1038/jid.2013.131, indexed in Pubmed: 23492918. 
15. Egeberg A, Skov L, Joshi AA, et al. The relationship between duration of psoriasis, vascular inflammation, and cardiovascular events. J Am Acad Dermatol. 2017; 77(4): 650-656.e3, doi: 10.1016/j.jaad.2017.06.028, indexed in Pubmed: 28826925.

16. Reich K. The concept of psoriasis as a systemic inflammation: implications for disease management. J Eur Acad Dermatol Venereol. 2012; 26(Suppl 2): 3-11, doi: 10.1111/j.1468-3083.2011.04410.x, indexed in Pubmed: 22356630
17. Abuabara K, Azfar RS, Shin DB, et al. Cause-specific mortality in patients with severe psoriasis: a population-based cohort study in the U.K. $\mathrm{Br} J$ Dermatol. 2010; 163(3): 586-592, doi: 10.1111/j.1365-2133.2010.09941.x, indexed in Pubmed: 20633008.

18. Ntusi NAB, Francis JM, Sever E, et al. Anti-TNF modulation reduces myocardial inflammation and improves cardiovascular function in systemic rheumatic diseases. Int J Cardiol. 2018; 270: 253-259, doi: 10.1016/j. ijcard.2018.06.099, indexed in Pubmed: 30017519. 